\title{
A Wideband Dual-Polarized Antenna Using Planar Quasi-Open-Sleeve Dipoles for Base Station Applications
}

\author{
Guan-xi Zhang, Li Sun, and Bao-hua Sun \\ National Laboratory of Antennas and Microwave Technology, Xidian University, Xi'an, Shaanxi 710071, China \\ Correspondence should be addressed to Guan-xi Zhang; gxzhang_xidian@126.com
}

Received 2 May 2015; Accepted 27 July 2015

Academic Editor: Herve Aubert

Copyright (c) 2015 Guan-xi Zhang et al. This is an open access article distributed under the Creative Commons Attribution License, which permits unrestricted use, distribution, and reproduction in any medium, provided the original work is properly cited.

\begin{abstract}
A wideband dual-polarized antenna for WLAN, WiMAX, and LTE base station applications is presented in this paper. The proposed antenna consists of two pairs of orthogonal planar quasi-open-sleeve dipoles along the centerlines, a balanced feeding structure and a square ground plane. The planar quasi-open-sleeve dipole comprises a pair of bowtie-shaped planar dipoles with two parallel curve parasitic elements. The introduced parallel curve parasitic elements change the path of the current of the original bowtieshaped planar dipoles at high frequencies and hence wideband characteristic is achieved. Two pairs of the planar quasi-open-sleeve dipoles placed orthogonally further broaden the bandwidth of the antenna with dual-polarization characteristics. The proposed antenna achieves a $10-\mathrm{dB}$ return loss bandwidth from 2.32 to $4.03 \mathrm{GHz}(53.9 \%$ bandwidth) using the planar quasi-open-sleeve dipole structures. The isolation between the two ports remains more than $32 \mathrm{~dB}$ in the whole bandwidth. Measured results show that the proposed antenna keeps the cross-polarization under $-33 \mathrm{~dB}$ and the front-to-back ratio better than $15 \mathrm{~dB}$ in the operating band. The antenna has an area of $0.3 \lambda \times 0.3 \lambda$ at $2.32 \mathrm{GHz}$ making it easy to be extended to an array element.
\end{abstract}

\section{Introduction}

Dual-polarized antennas have obtained wide applications in various mobile communications such as WLAN, WiMAX, and LTE base stations. In order to cover these bands, dualpolarized antennas are required to have a broad bandwidth. In addition, high port-to-port isolation and low cross-polarization level are also required for base station antennas. Designing the dual-polarized antennas that meet these requirements is a challenging work.

Several techniques have been developed to improve the bandwidth [1-4]. A feeding structure of aperture coupling has applied in [1-3] achieving an impendence bandwidth about $20 \%$. The feeding structure can also improve the port isolation. A stacked patch is introduced that obtains an impedance bandwidth over 33\% in [4]. However, all these antennas have the disadvantages of high cross-polarization levels due to the transverse currents of high-order modes.

Several techniques have been developed to improve the port isolation and the cross-polarization [5-10]. The antennas in [5-7] are symmetrically excited by a differential feed system with a phase difference of $180^{\circ}$. This feeding structure can improve the cross-polarization by suppressing the higherorder modes. A balanced feeding structure with two shorting pins and two feeding coax lines is introduced in [8-10] to improve the isolation. The antenna in [10] also achieves a wide impendence bandwidth of $45.8 \%$.

The cylindrical open-sleeve dipole contains a dipole with two closely spaced parasitic elements or sleeves [11]. This kind of antennas takes the merits of broadband characteristic [12]. In recent decades, the planar open-sleeve dipole has been investigated in many literatures [13-15]. These antennas are conformal and can be used to achieve a broadband/dual-band characteristic. The end-loaded planar open-sleeve dipole antenna in [13] achieves a wide impendence bandwidth of $50 \%$. Unfortunately, these antennas are single linear polarization. The planar open-sleeve dipole has not been used as dual-polarized antennas.

In this paper, a wideband dual-polarized antenna with planar quasi-open-sleeve dipole structures is presented. A pair of bowtie-shaped planar dipoles and two parallel curve parasitic elements constitute the planar quasi-open-sleeve dipole structures with wideband characteristics. Better wideband and dual-polarization characteristics are achieved by 


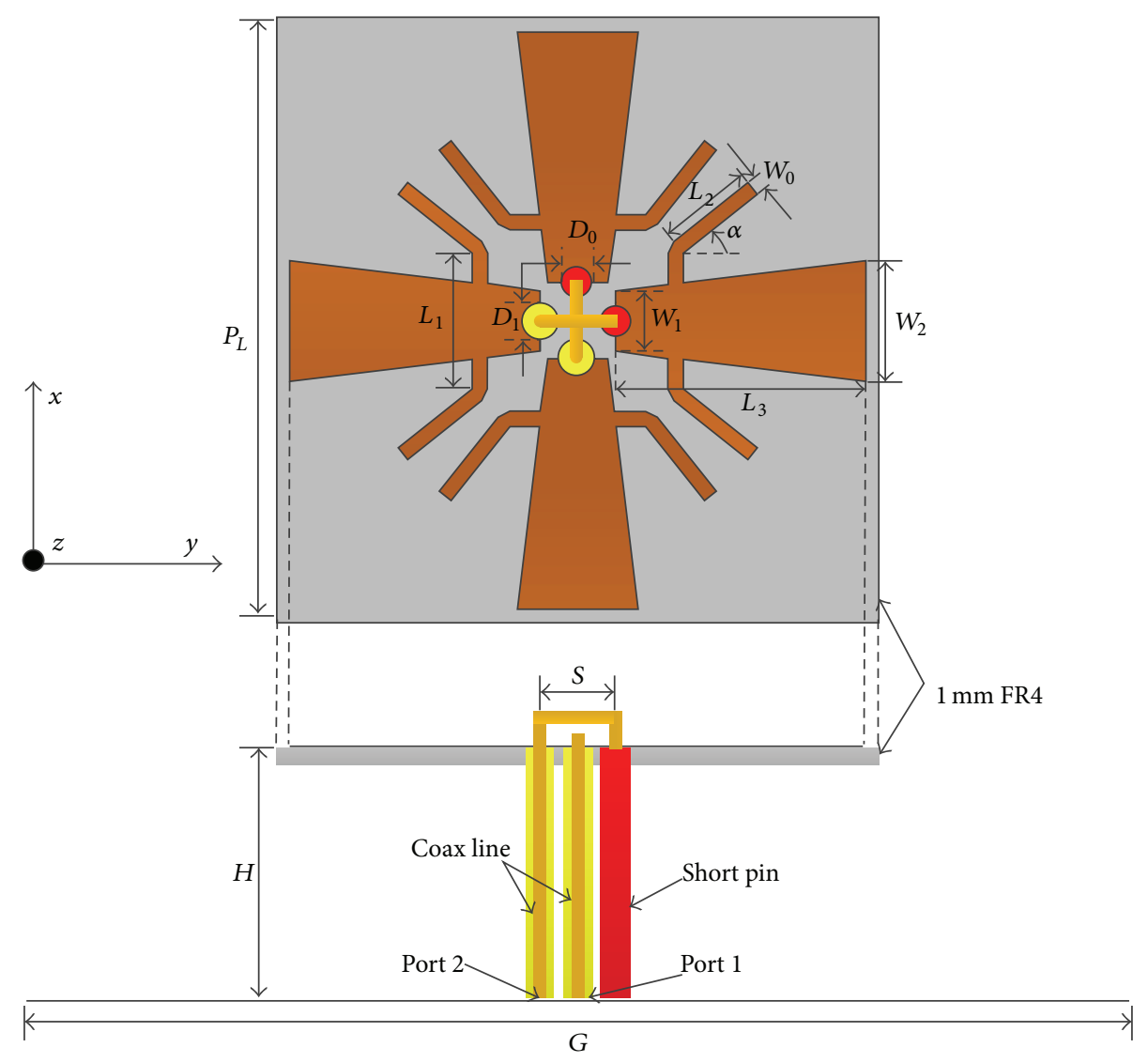

FIGURE 1: Geometry of the proposed antenna with the detailed design parameters.

orthogonally placing two pairs of planar quasi-open-sleeve dipoles. The proposed antenna achieves a $10-\mathrm{dB}$ return loss bandwidth from 2.32 to $4.03 \mathrm{GHz}$ (53.9\% bandwidth) using the planar quasi-open-sleeve dipole structures. By introducing two feeding coax lines with two shorting pins as the balanced feeding structure, the isolation between the two ports remains more than $32 \mathrm{~dB}$ in the operating band. Experimental results of the constructed prototype show good crosspolarization performance. Details of the antenna design and both theoretical and experimental results are presented and discussed in the following sections.

\section{Antenna Geometry}

The geometry of the proposed dual-port and dual-polarized wideband planar dipole is given in Figure 1, with detailed dimensions shown in Table 1 . The antenna was constructed by printing it on RF4 substrate, with a thickness of $1 \mathrm{~mm}$, a relative permittivity of 4.4 , and an area of $40 \mathrm{~mm} \times 40 \mathrm{~mm}$. In the design, the bowtie-shaped planar dipoles unite the curve parasitic elements, printed to the surface of the structure. A balanced feeding structure, shown in Figure 1, consists of two shorting pins and two feeding coax cables. The two coax lines are placed along the centerline of the radiating patch and are located at a distance of $S / 2$ from the center of the patch. The outer part of the feeding coax cable is connected to one arm of the dipole (yellow circle in Figure 1) and the inner
TABLE 1: Dimensions of the proposed antenna.

\begin{tabular}{lcccccc}
\hline Parameters & $G$ & $H$ & $P_{L}$ & $L_{1}$ & $L_{2}$ & $L_{3}$ \\
\hline Values/mm & 100 & 27.4 & 40 & 9 & 4.9 & 17.2 \\
\hline Parameters & $W_{0}$ & $W_{1}$ & $W_{2}$ & $S$ & $D_{0}$ & $D_{1}$ \\
\hline Values/mm & 1 & 4 & 8 & 5 & 2 & 2.4 \\
\hline Parameters & $\alpha$ & & & & & \\
\hline Values/degree & 53 & & & & &
\end{tabular}

part connected to the other arm of the dipole (red circle in Figure 1). With two shorting pins connecting to the inner part of the feeding coax, good isolation between the two ports is achieved.

\section{Principle of Operation}

To better understand the excitation behavior of the proposed antenna, Figure 2 shows the evolution steps of the proposed antenna design. Antenna A is shown in Figure 2(a), which is composed of a class planar bowtie-shaped dipole operating at $2.5 \mathrm{GHz}$. In order to verify the impact of two curve parasitic elements on the planar bowtie-shaped dipole antenna, two curve parasitic elements perpendicular to the axis of the bowtie-shaped dipole are added symmetrically to both sides of the antenna, as shown in Figure 2(b). In order 


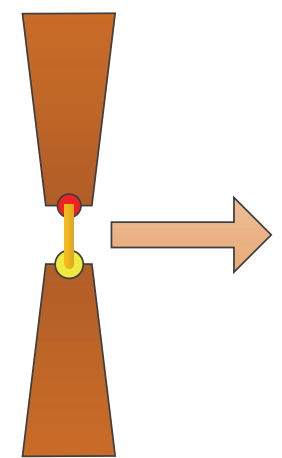

Antenna A

(a)

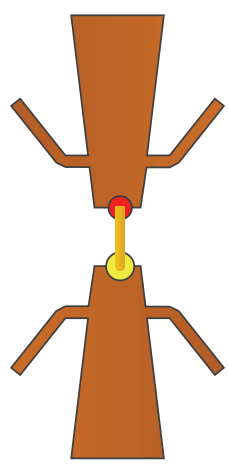

Antenna B

(b)

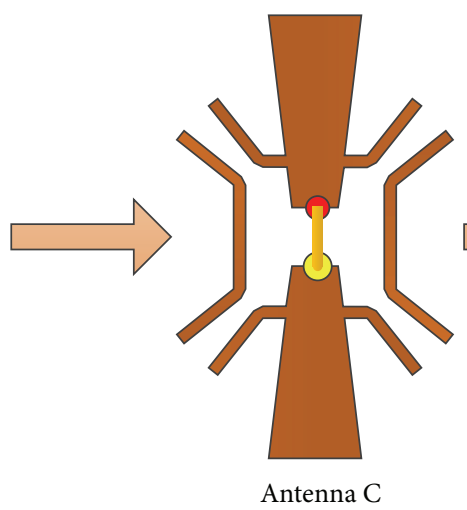

(c)

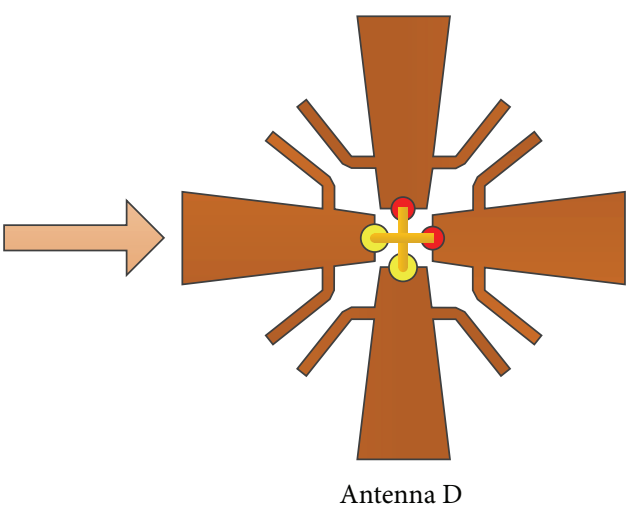

(d)

FIGURE 2: The evolution steps of the proposed antenna configuration: (a) a planar bowtie-shaped dipole (Antenna A), (b) a planar bowtieshaped dipole with two curve parasitic elements (Antenna B), (c) a planar bowtie-shaped dipole with four curve parasitic elements (Antenna C), and (d) the proposed antenna (Antenna D).

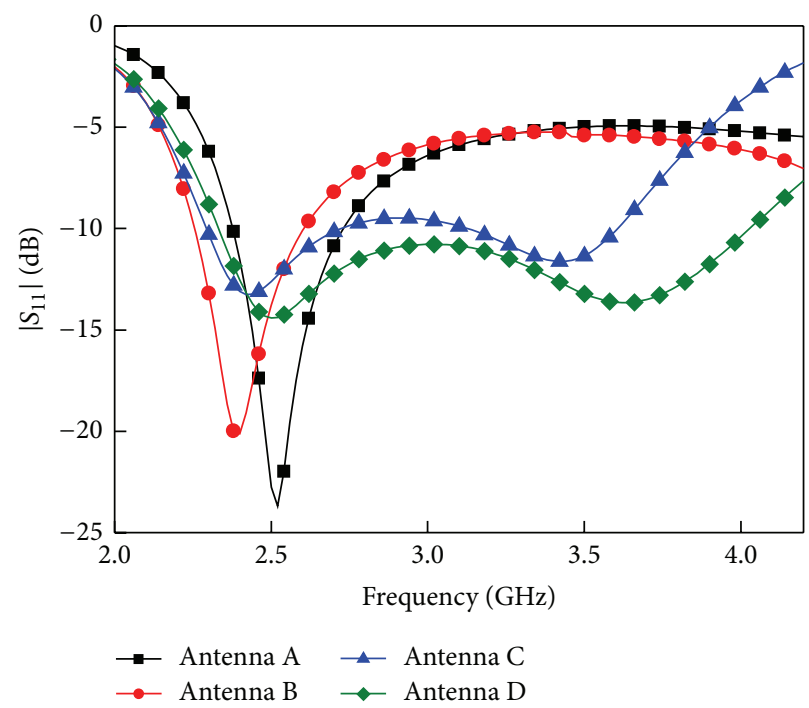

FIGURE 3: Simulated $|S 11|$ performances of the antennas.

to widen the bandwidth of the bowtie-shaped dipole, two curve parasitic elements parallel to the axis of the bowtieshaped dipole are added symmetrically to both sides of the antenna. Antenna C shown in Figure 2(c) is designed. Finally, for obtaining double linear polarizations, two pairs of planar quasi-open-sleeve dipoles which are perpendicular are printed on the substrate, as the proposed antenna (Antenna D) presented in Figure 2(d).

Figure 3 illustrates the $|S 11|$ variation of Antenna A, Antenna B, Antenna C, and Antenna D. It can be seen that Antenna A shows good impedance matching at $2.5 \mathrm{GHz}$. By adding two perpendicular curve parasitic elements, the resonance frequency shifts slightly from $2.5 \mathrm{GHz}$ to $2.4 \mathrm{GHz}$. It is noticed that the perpendicular curve parasitic element does not destroy the resonance characteristic of Antenna A. By adding another two parallel curve parasitic elements on Antenna $\mathrm{B}$, a new upper resonant frequency around $4 \mathrm{GHz}$ is generated without changing the resonance characteristics at 2.4 GHz. Antenna $\mathrm{C}$ shows broadband characteristics with lower resonant frequency at $2.4 \mathrm{GHz}$ weakened. Finally, by placing two pairs of planar quasi-open-sleeve dipoles perpendicularly, the proposed antenna (Antenna D) achieves good advantage of broadband characteristics with both lower and upper resonant frequencies enhanced.

To further validate the wideband characteristics, the current distributions of the proposed dual-polarized antenna at $2.5 \mathrm{GHz}$ and $3.6 \mathrm{GHz}$ are depicted in Figure 4 when Port 2 is excited. It is obvious that the current mainly flows along the horizontal bowtie-shaped dipole at $2.5 \mathrm{GHz}$. The bowtie shaped structure diminished the dimension of the antenna while the introduced curve parasitic elements have little influence on the current distributions at $2.5 \mathrm{GHz}$. Thus, the operation for lower band is achieved by the horizontal bowtieshaped dipole. As shown in Figure 4(b), the introduced curve parasitic elements change the patch of the current with the currents mainly flowing along the four curve elements instead 


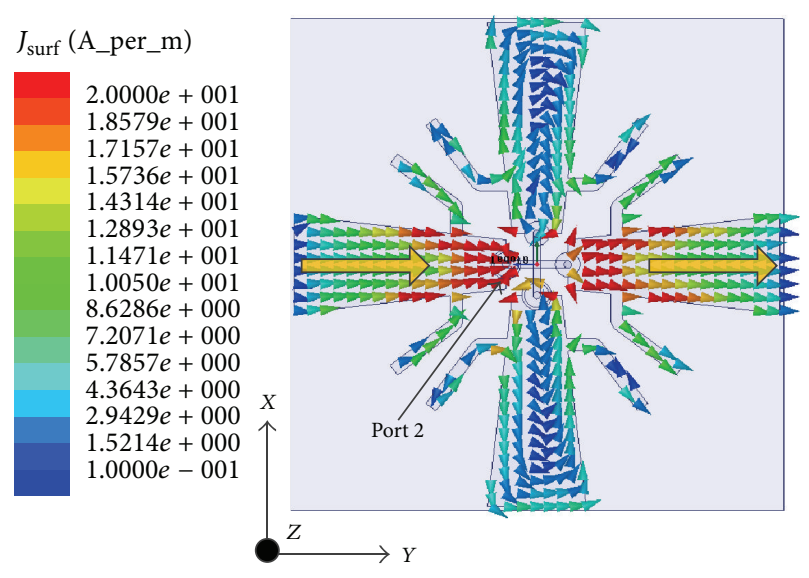

(a)

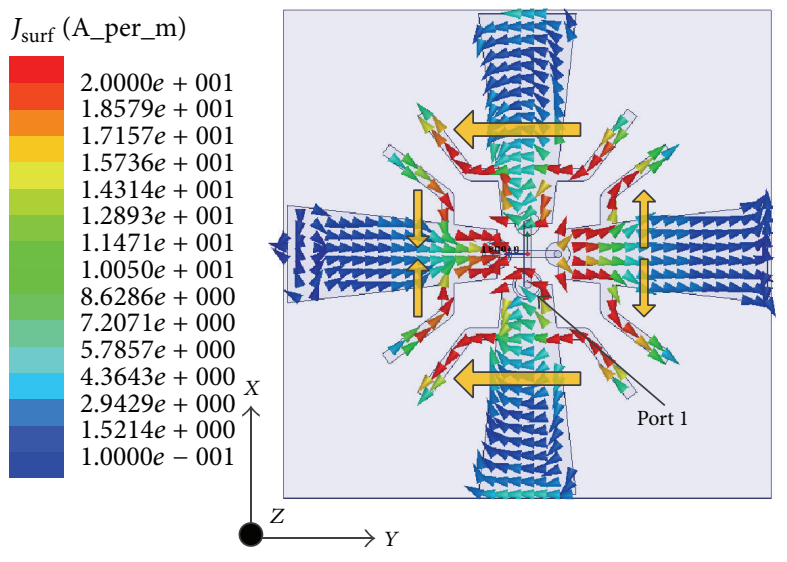

(b)

FIgURE 4: Current distribution of the proposed antenna at (a) $2.5 \mathrm{GHz}$ and (b) $3.6 \mathrm{GHz}$ for Port 2.

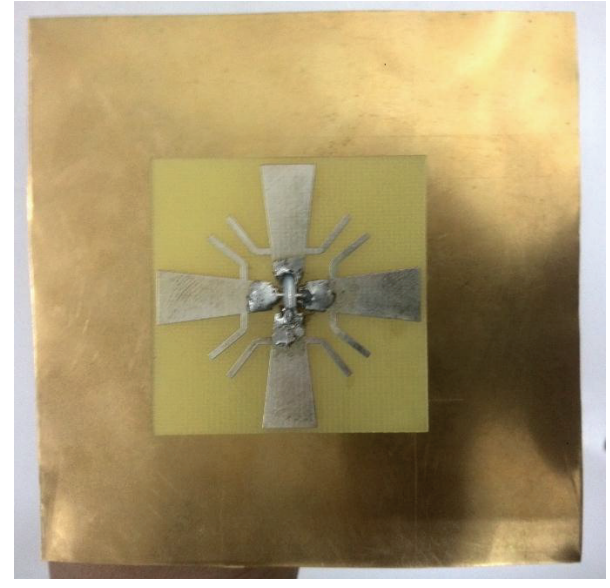

FIgURE 5: Photograph of the proposed antenna.

of the bowtie-shaped dipole at $3.6 \mathrm{GHz}$. Furthermore, the currents on the two horizontal curve parasitic elements are in phase, while the currents on the two vertical curve parasitic elements are out of phase. Thus, the current along the $y$-axis is enhanced while the current along $x$-axis is offset. Therefore, the proposed antenna achieved resonating at $3.6 \mathrm{GHz}$ with the same polarization as the lower resonance frequency.

\section{Simulated and Measured Results}

The proposed antenna is designed, constructed, and tested. Figure 5 shows the photograph of the proposed antenna. The simulated and measured reflection coefficients of each port of the proposed antenna are shown in Figure 6. It can be seen that the antenna exhibits a measured $-10 \mathrm{~dB}$ impedance bandwidth of $1730 \mathrm{MHz}$ and $1740 \mathrm{MHz}$ for Port 1 and Port 2 , respectively, which can cover WLAN (2400-2484 MHz), LTE (2500-2690 MHz), and WiMAX (3300-3800 MHz). The measured S11/S22 characteristics shown in Figure 6 imply that the mutual coupling of parasitic elements is greater than

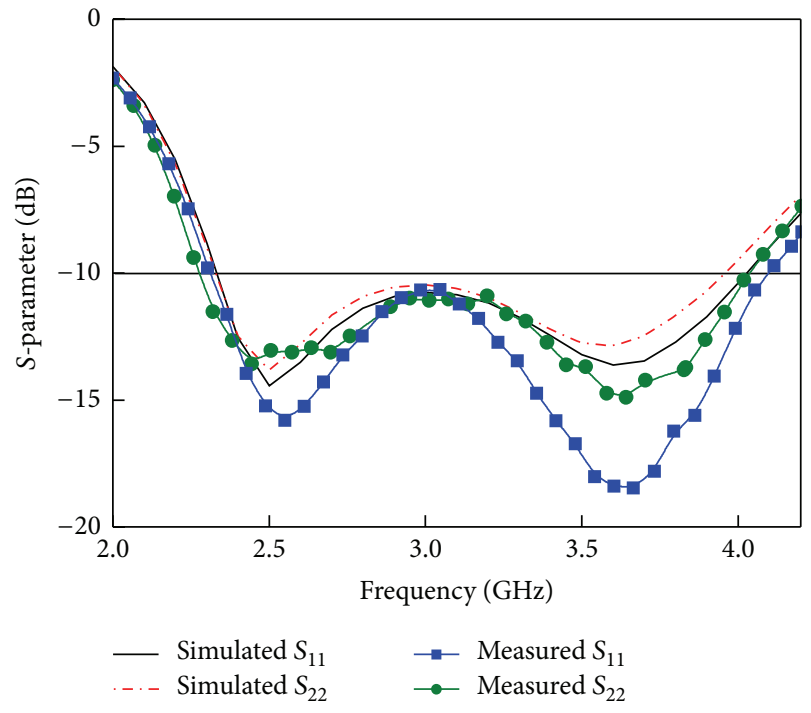

FIGURE 6: Measured and simulated S11/S22 of the proposed antenna.

expected from the simulations. Besides, the asymmetry of S11 and S22 results from the coupling effects in feeding structure. The simulated and measured S21 between the two ports are shown in Figure 7. It is observed that the simulated port isolation and measured port isolation are better than $33 \mathrm{~dB}$ over the frequency band from $2.32 \mathrm{GHz}$ to $4.03 \mathrm{GHz}$. The deterioration of isolation in higher frequencies is caused by the effect of parasitic elements and its mutual coupling shown in Figure 4.

Measured radiation patterns in both of E- and H-plane at $2.5 \mathrm{GHz}, 3 \mathrm{GHz}$, and $3.5 \mathrm{GHz}$ for Port 1 are illustrated in Figures 8 and 9 , respectively. Owing to the symmetrical characteristic of the proposed antenna, the radiation patterns of the proposed antenna have only a slight difference between the two ports. Thus, we just show the measured radiation results of Port 1 . When one port is measured, the other port is connected to the matched load. 


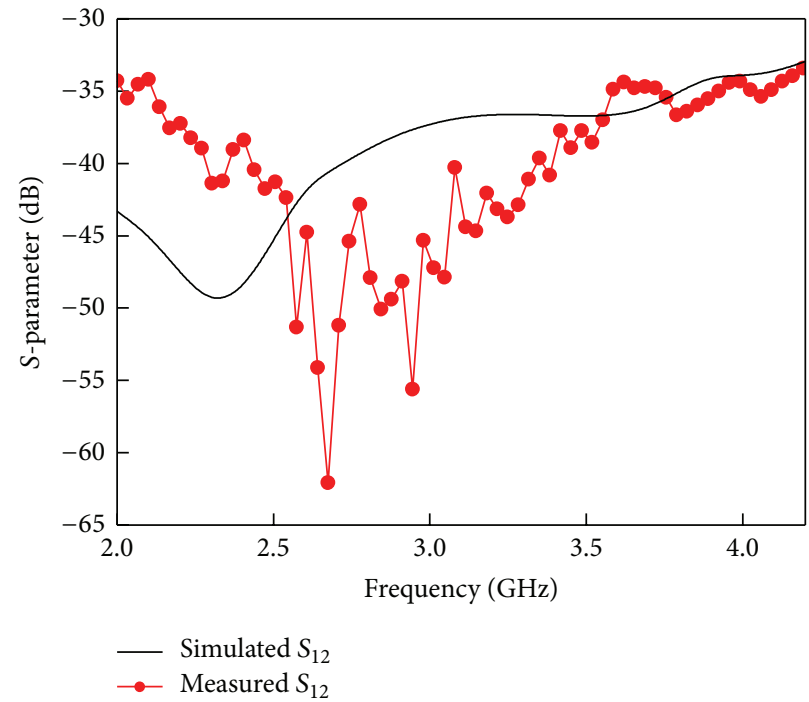

FIGURE 7: Measured and simulated S21 of the proposed antenna.

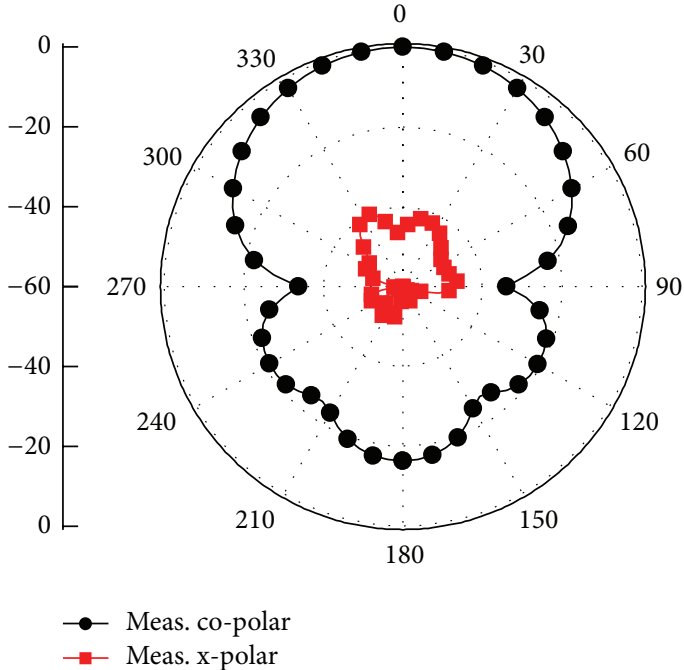

(a)

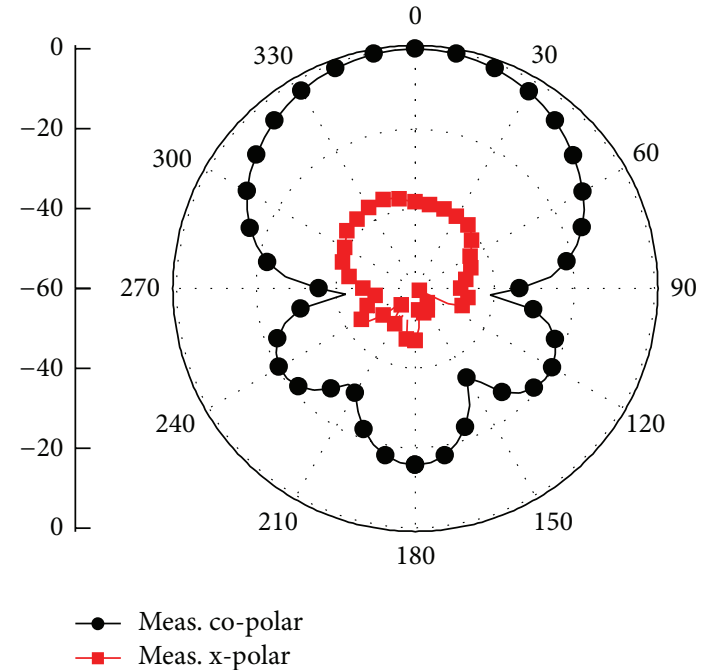

(b)

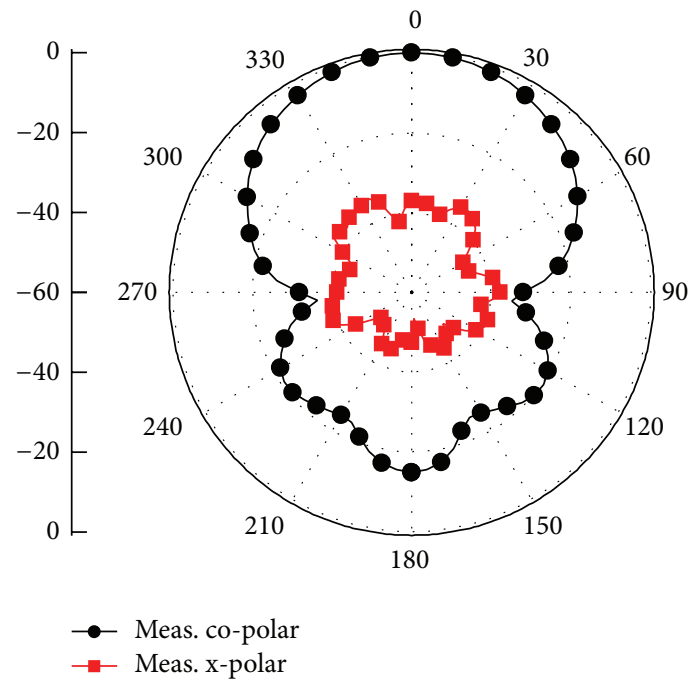

(c)

Figure 8: Measured radiation patterns for E-plane (xoz plane) at (a) $2.5 \mathrm{GHz}$, (b) $3 \mathrm{GHz}$, and (c) $3.5 \mathrm{GHz}$ for Port 1. 


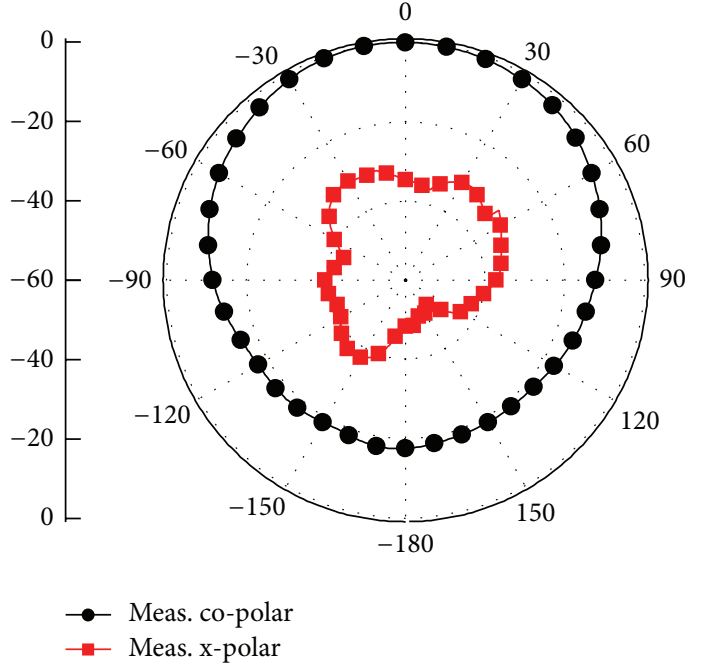

(a)

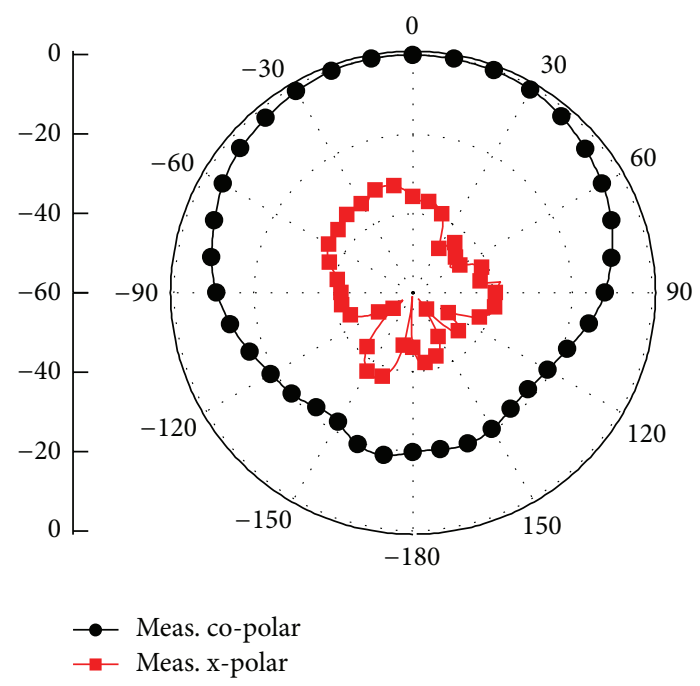

(b)

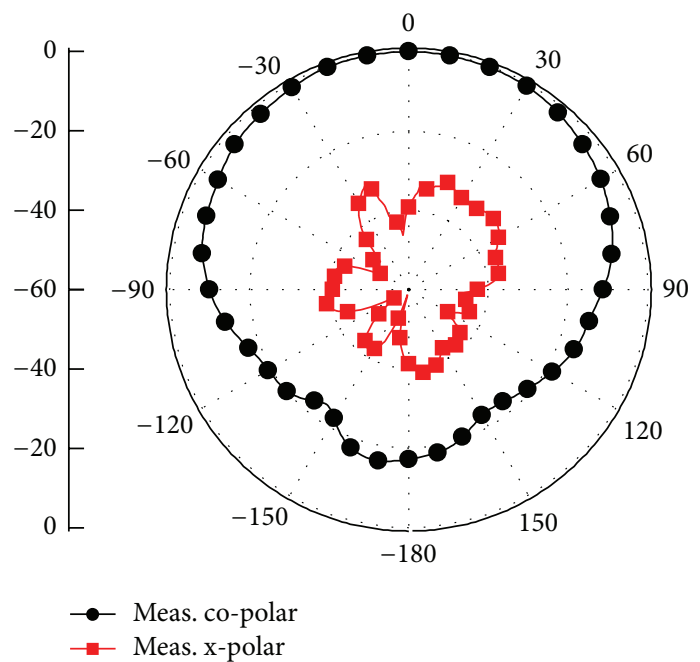

(c)

Figure 9: Measured radiation patterns for H-plane (yoz plane) at (a) $2.5 \mathrm{GHz}$, (b) $3 \mathrm{GHz}$, and (c) $3.5 \mathrm{GHz}$ for Port 1.

It can be seen that the measured cross-polarization levels within the main lobe are less than $-32 \mathrm{~dB}$ in both $\mathrm{E}$ - and $\mathrm{H}$ plane for the two ports, while the front-to-back ratio remains over $15 \mathrm{~dB}$ due to small metallized ground plane. The simulated and measured gains of the antenna are shown in Figure 10. The gain for the two ports ranges from $7.2 \mathrm{dBi}$ to $7.8 \mathrm{dBi}$ within the operation frequency band.

\section{Conclusion}

In this paper, a wideband dual-polarized antenna with low cross-polarization and high isolation is designed by using two pairs of orthogonal planar quasi-open-sleeve dipoles. The introduced curve parasitic elements change the path of the current distributions at higher resonant frequencies but achieve the consistent polarization in the whole operating band. The proposed antenna is excited by two feeding coax lines and shorted by two pins as the balancing feeding structure. A prototype with center frequency of $3.1 \mathrm{GHz}$ was designed,

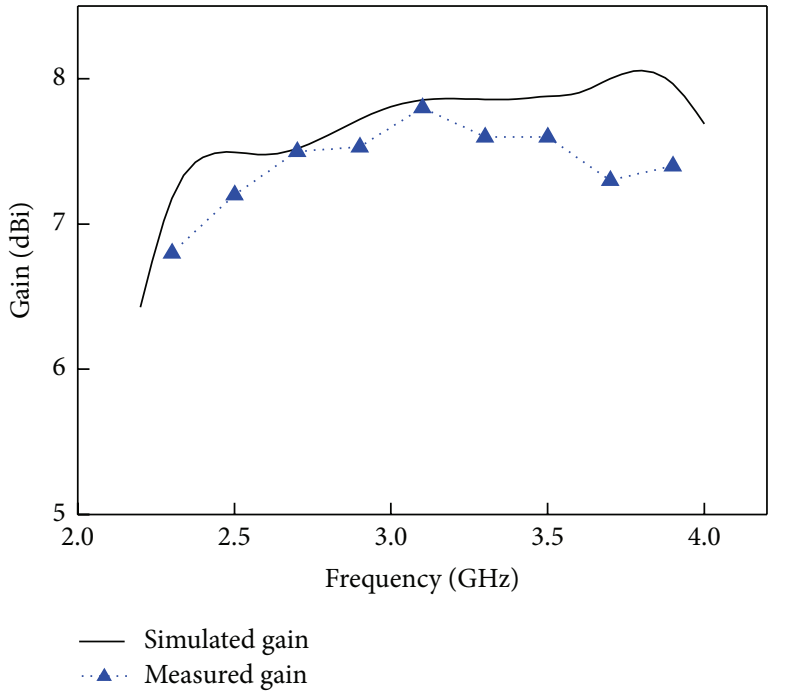

FIGURE 10: Measured and simulated gain of the proposed antenna. 
fabricated, and tested. The proposed antenna achieves a $53.9 \%$ impedance bandwidth for $10-\mathrm{dB}$ return loss $(2.32 \mathrm{GHz}-$ $4.03 \mathrm{GHz}$ ), a $-32 \mathrm{~dB}$ cross-polarization level for both polarization with low back radiation, a $33 \mathrm{~dB}$ port isolation, and an average gain of $7.5 \mathrm{dBi}$ in the whole bandwidth. Work is in progress to realize a $2 \times 2$ planar array to be used as a small cell antenna for mobile communication base stations.

\section{Conflict of Interests}

The authors declare that there is no conflict of interests regarding the publication of this paper.

\section{References}

[1] S. K. Padhi, N. C. Karmakar Sr., C. L. Law, and S. Aditya, "A dual polarized aperture coupled circular patch antenna using a C-shaped coupling slot," IEEE Transactions on Antennas and Propagation, vol. 51, no. 12, pp. 3295-3298, 2003.

[2] S. Gao, L. W. Li, M. S. Leong, and T. S. Yeo, "A broad-band dualpolarized microstrip patch antenna with aperture coupling," IEEE Transactions on Antennas and Propagation, vol. 51, no. 4, pp. 898-900, 2003.

[3] T.-W. Chiou and K.-L. Wong, "Broad-band dual-polarized single microstrip patch antenna with high isolation and low cross polarization," IEEE Transactions on Antennas and Propagation, vol. 50, no. 3, pp. 399-401, 2002.

[4] A. A. Serra, P. Nepa, G. Manara, G. Tribellini, and S. Cioci, "A wide-band dual-polarized stacked patch antenna," IEEE Antennas and Wireless Propagation Letters, vol. 6, pp. 141-143, 2007.

[5] C.-Y. Sim, C.-C. Chang, and J.-S. Row, "Dual-feed dualpolarized patch antenna with low cross polarization and high isolation," IEEE Transactions on Antennas and Propagation, vol. 57, no. 10, pp. 3405-3409, 2009.

[6] H. Wong, K.-L. Lau, and K.-M. Luk, "Design of dual-polarized L-probe patch antenna arrays with high isolation," IEEE Transactions on Antennas and Propagation, vol. 52, no. 1, pp. 45-52, 2004.

[7] T.-W. Chiou and K.-L. Wong, "A compact dual-band dualpolarized patch antenna for $900 / 1800-\mathrm{MHz}$ cellular systems," IEEE Transactions on Antennas and Propagation, vol. 51, no. 8, pp. 1936-1940, 2003.

[8] B. Li, Y.-Z. Yin, Y. Zhao, Y. Ding, and R. Zou, "Dual-polarised patch antenna with low cross-polarisation and high isolation for WiMAX applications," Electronics Letters, vol. 47, no. 17, pp. $952-$ 953, 2011.

[9] S.-G. Zhou, P.-K. Tan, and T.-H. Chio, "Low-profile, wideband dual-polarized antenna with high isolation and low cross polarization," IEEE Antennas and Wireless Propagation Letters, vol. 11, pp. 1032-1035, 2012.

[10] B. Li, Y.-Z. Yin, W. Hu, Y. Ding, and Y. Zhao, "Wideband dualpolarized patch antenna with low cross polarization and high isolation," IEEE Antennas and Wireless Propagation Letters, vol. 11, pp. 427-430, 2012.

[11] W. L. Stutzman and G. A. Thiele, Antenna Theory and Design, Wiley, New York, NY, USA, 2nd edition, 1998.

[12] J. L. Wong and H. E. King, "Broadband characteristics of an open-sleeve dipole," in Proceedings of the Antennas and Propagation Society International Symposium, pp. 332-335, IEEE, 1972.
[13] T. G. Spence and D. H. Werner, "A novel miniature broadband/multiband antenna based on an end-loaded planar opensleeve dipole," IEEE Transactions on Antennas and Propagation, vol. 54, no. 12, pp. 3614-3620, 2006.

[14] G. A. Evtioushkine, J. W. Kim, and K. S. Han, "Very wideband printed dipole antenna array," Electronics Letters, vol. 34, no. 24, pp. 2292-2293, 1998.

[15] T. G. Spence, D. H. Werner, and R. D. Groff, "Genetic algorithm optimization of some novel broadband and multiband microstrip antennas," in Proceedings of the IEEE Antennas and Propagation Society Symposium, pp. 4408-4411, June 2004. 

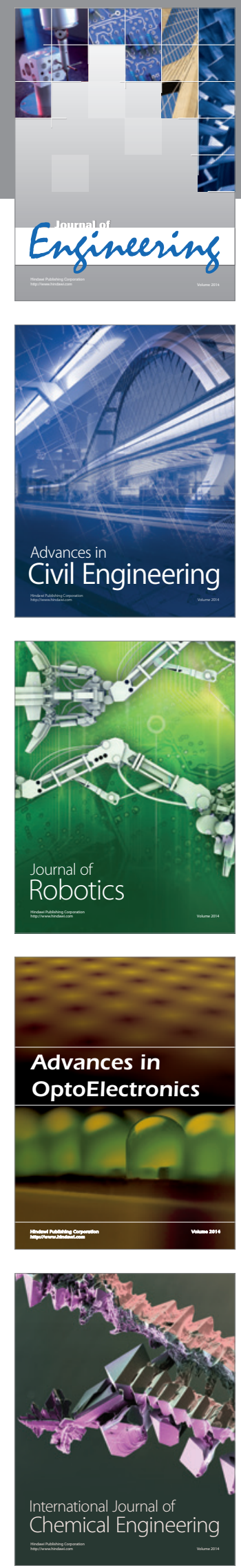

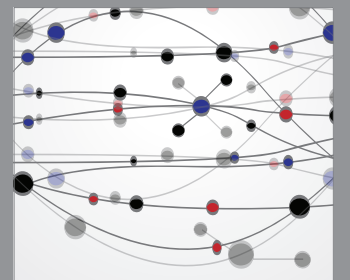

The Scientific World Journal
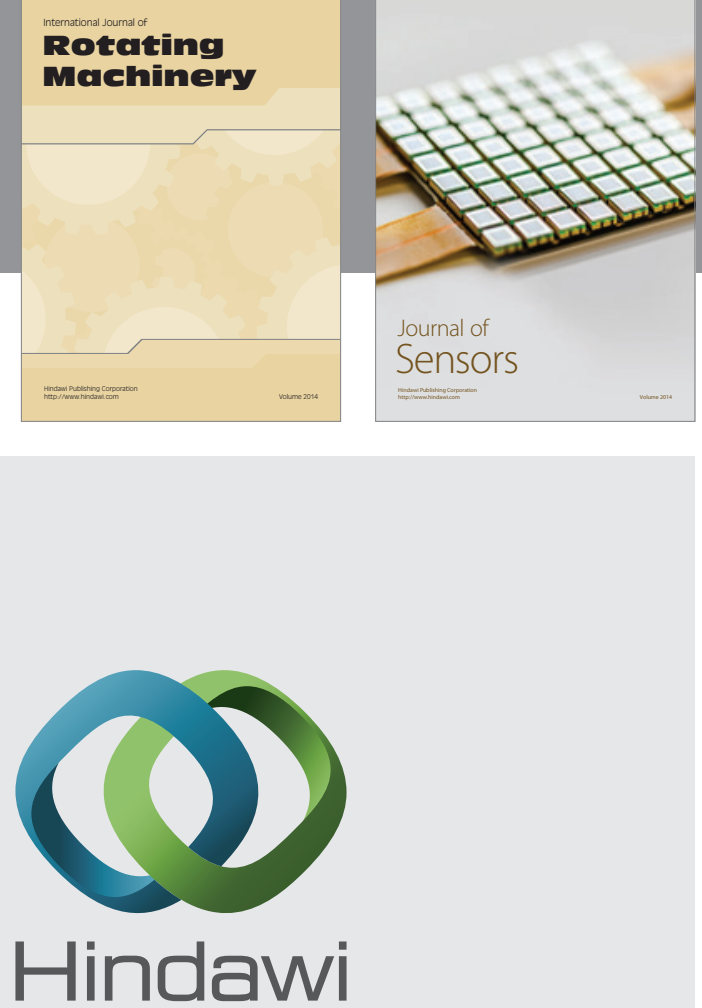

Submit your manuscripts at http://www.hindawi.com
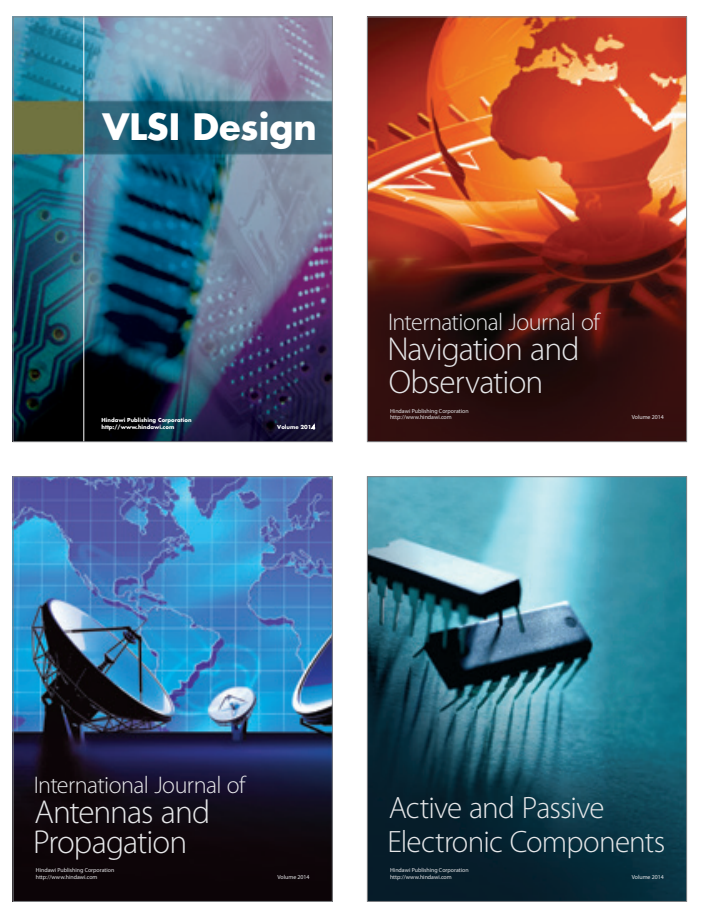
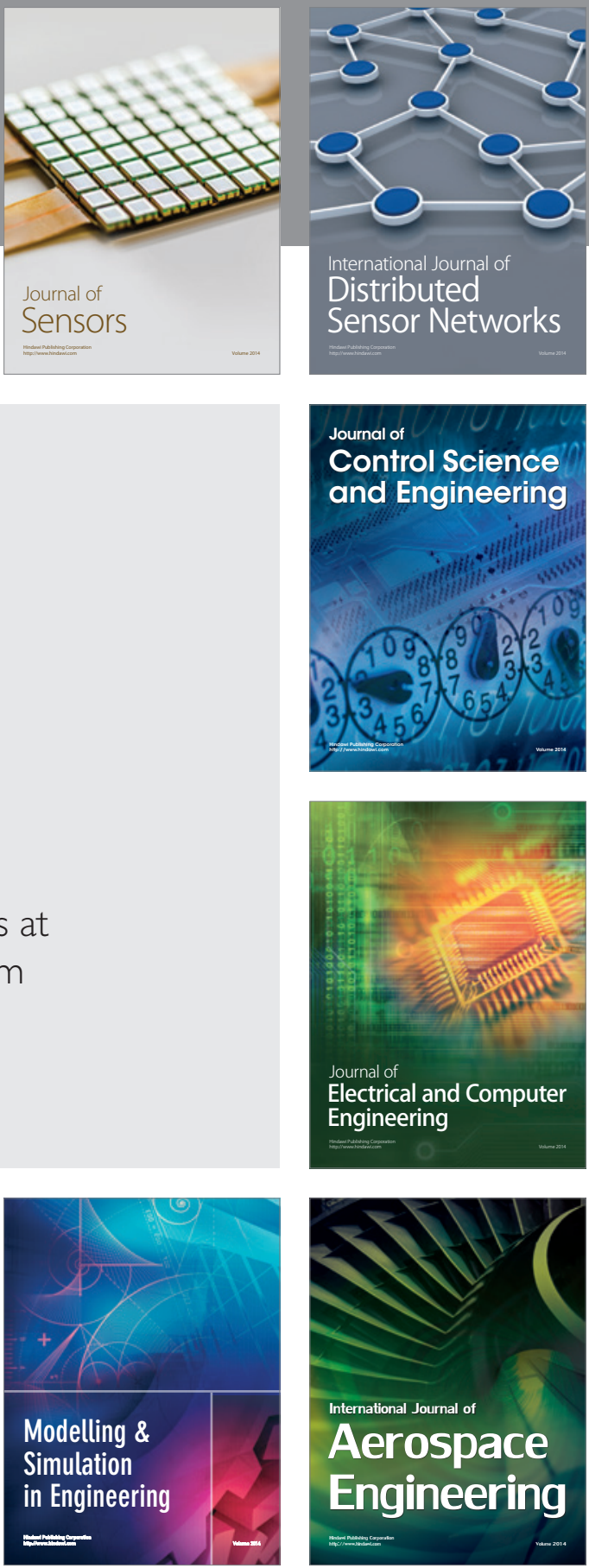

Journal of

Control Science

and Engineering
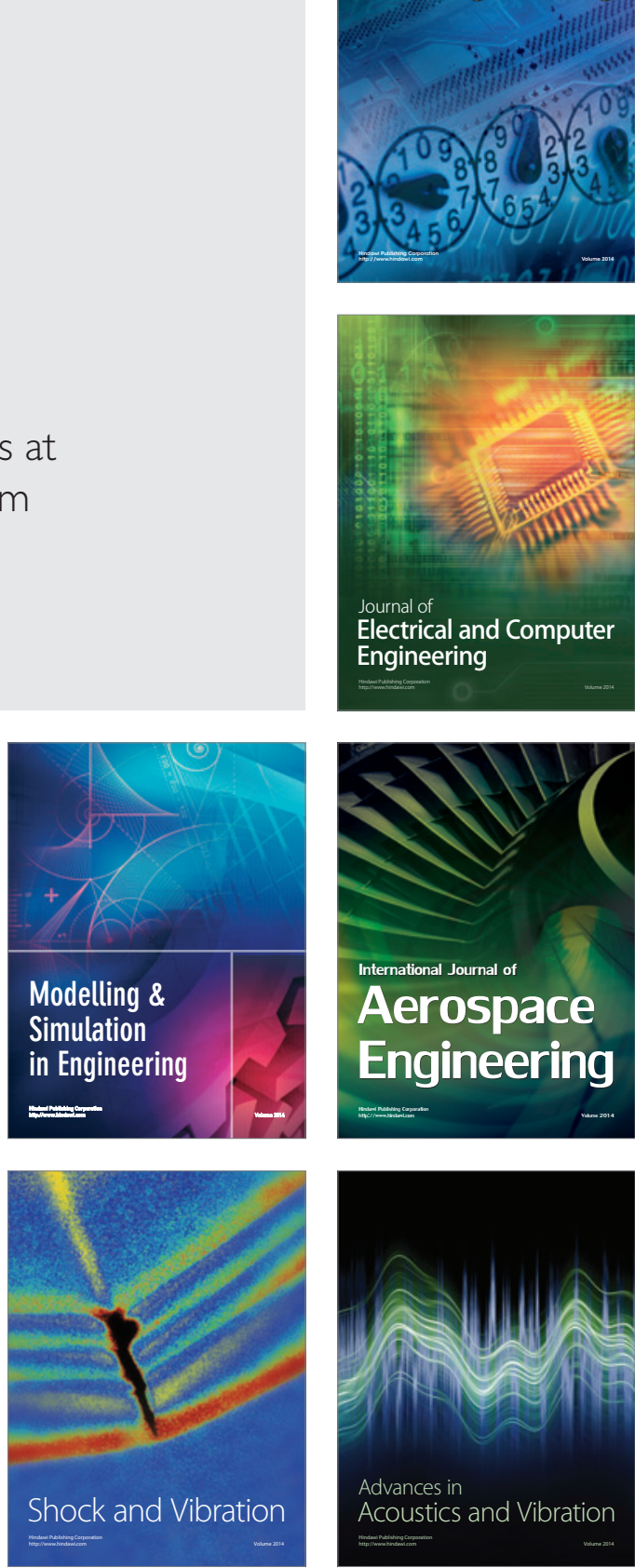